\title{
MATRIX INEQUALITIES INCLUDING GRAND FURUTA INEQUALITY VIA KARCHER MEAN
}

\author{
Masatoshi ITO
}

Abstract. In our previous paper, we have shown a generalization of Furuta inequality via Karcher mean (Riemannian mean) by using Yamazaki's results which are generalizations of Ando-Hiai inequality and related ones. In this paper, we shall show a generalization of grand Furuta inequality as an extension of our previous result.

Mathematics subject classification (2010): Primary 15A45, 47A63. Secondary 15B48, 47A64. inequality.

Keywords and phrases: Positive definite matrix, Karcher mean, Furuta inequality and grand Furuta

\section{REFERENCES}

[1] T. ANDo AND F. HiAI, Log majorization and complementary Golden-Thompson type inequalities, Linear Algebra Appl., 197, 198 (1994), 113-131.

[2] T. Ando, C. K. Li And R. Mathias, Geometric means, Linear Algebra Appl., 385 (2004), 305334.

[3] R. Bhatia, Positive definite matrices, Princeton Series in Applied Mathematics, Princeton University Press, Princeton, NJ, 2007.

[4] R. Bhatia And J. Holbrook, Riemannian geometry and matrix geometric means, Linear Algebra Appl., 413 (2006), 594-618.

[5] D. A. Bini, B. Meini AND F. Poloni, An effective matrix geometric mean satisfying the Ando-LiMathias properties, Math. Comp., 79 (2010), 437-452.

[6] M. FUJII, Furuta's inequality and its mean theoretic approach, J. Operator Theory, 23 (1990), 67-72.

[7] M. FujiI, T. FurutA AND E. KAMEI, Furuta's inequality and its application to Ando's theorem, Linear Algebra Appl., 179 (1993), 161-169.

[8] M. FujiI AND E. KAMEI, Mean theoretic approach to the grand Furuta inequality, Proc. Amer. Math. Soc., 124 (1996), 2751-2756.

[9] T. FurutA, $A \geqslant B \geqslant 0$ assures $\left(B^{r} A^{p} B^{r}\right)^{1 / q} \geqslant B^{(p+2 r) / q}$ for $r \geqslant 0, p \geqslant 0, q \geqslant 1$ with $(1+2 r) q \geqslant$ $p+2 r$, Proc. Amer. Math. Soc., 101 (1987), 85-88.

[10] T. FURUTA, An elementary proof of an order preserving inequality, Proc. Japan Acad. Ser. A Math. Sci., 65 (1989), 126.

[11] T. FuRUTA, Applications of order preserving operator inequalities, Oper. Theory Adv. Appl., 59 (1992), 180-190.

[12] T. FURUTA, Extension of the Furuta inequality and Ando-Hiai log-majorization, Linear Algebra Appl., 219 (1995), 139-155.

[13] T. FuRUTA, Simplified proof of an order preserving operator inequality, Proc. Japan Acad. Ser. A Math. Sci., 74 (1998), 114.

[14] M. ITo, Matrix inequalities including Furuta inequality via Riemannian mean of n-matrices, J. Math. Inequal., 6 (2012), 481-491.

[15] S. IZUMino AND N. NAKAmuRA, Weighted geometric means of positive operators, Kyungpook Math. J., 50 (2010), 213-228.

[16] E. KAMEI, A satellite to Furuta's inequality, Math. Japon., 33 (1988), 883-886.

[17] J. D. LAWSON AND Y. LiM, Monotonic properties of the least squares mean, Math. Ann., 351 (2011), 267-279. 
[18] M. MOAKHER, A differential geometric approach to the geometric mean of symmetric positive-definite matrices, SIAM J. Matrix Anal. Appl., 26 (2005), 735-747.

[19] K. Tanahashi, Best possibility of the Furuta inequality, Proc. Amer. Math. Soc., 124 (1996), 141146.

[20] K. TAnAhashi, The best possibility of the grand Furuta inequality, Proc. Amer. Math. Soc., 128 (2000), 511-519.

[21] T. YAMAZAKI, The Riemannian mean and matrix inequalities related to the Ando-Hiai inequality and chaotic order, Oper. Matrices., 6 (2012), 577-588. 